\title{
¿Está contaminado el aire de la escuela? Una propuesta de indagación basada en la modelización para el aula de primaria
}

\author{
Èlia Tena
}

Dep. de Didàctica de la Matemàtica i les Ciències Experimentals y CRECIM.

UAB. Barcelona. España

[Recibido el 5 de marzo de 2021, aceptado el 30 de junio de 2021]

Diseñar secuencias de enseñanza y aprendizaje (SEA) desde un paradigma STEM con valores y que involucren al alumnado en prácticas científicas auténticas es un reto para los docentes. Por ello, en el siguiente artículo se describen tanto los principios de diseño como las actividades clave de una SEA que parte de la problemática de la contaminación atmosférica diseñada siguiendo un proceso iterativo. La SEA ha permitido a alumnado de 5ㅇ y 6 o curso de primaria: 1) construir ideas clave del modelo corpuscular de la materia escolar para esta edad partiendo de sus ideas, 2) involucrarse en una indagación genuina y 3) proponer soluciones justificadas para disminuir la contaminación alrededor de su escuela.

Palabras clave: STEM; indagación basada en la modelización; secuencia didáctica; contaminación; educación primaria

\section{Is our school air polluted? A model-based inquiry proposal for primary schools}

Designing values-based STEM teaching and learning sequences (TLS) that engage pupils in real scientific practices is not an easy task for teachers. This paper describes the design principles and main activities of a model-based inquiry TLS for fifth- and sixth-class primary school pupils focused on the phenomena of pollution. The results of the TLS showed that the experience allowed pupils to: 1) formulate key ideas about the corpuscular theory of matter at primary school level, 2) participate in a real school-based inquiry, and 3) design reasoned solutions to pollution in their area.

Keywords: STEM; modelling-based inquiry; teaching and learning sequence; pollution; primary school education

Para citar este artículo. Tena, È. (2021). ¿Está contaminado el aire de la escuela? Una propuesta de indagación basada en la modelización para el aula de primaria. Ápice. Revista de Educación Científica, 5(2), 87-97. DOI: https://doi.org/10.17979/arec.2021.5.2.7613

Contacto. elia.tena@uab.cat 


\section{Introducción}

Existe un número creciente y diverso de proyectos y propuestas para educación primaria que se enmarcan en un paradigma STEM (siglas en inglés de Ciencias, Tecnología, Ingeniería y Matemáticas) (Couso, 2017). De acuerdo con esta autora, que propone un marco basado en la idea de participación en las practicas científico-tecnológicas y los valores para la educación STEM, estas propuestas deberían perseguir la alfabetización del alumnado en este ámbito. Es decir, el alumnado debería:

\footnotetext{
"Ser capaz de identificar y aplicar tanto los conocimientos clave de las disciplinas STEM, como las formas de hacer, pensar, hablar y sentir de la ciencia, la ingeniería y la matemática, de forma más o menos integrada, para comprender, decidir y/o actuar delante de problemas complejos y/o construir soluciones creativas e innovadoras que aprovechen las sinergias personales y las tecnologías disponibles de forma crítica, reflexiva y con valores" (Couso, 2017, p. 24).
}

De acuerdo con la definición, el diseño de secuencias didácticas (SEA) dentro del marco STEM debe: (1) permitir al alumnado construir conocimiento profundo para actuar sobre problemáticas relevantes científica y socialmente, (2) involucrarlos en actividades auténticas y genuinas de las disciplinas y (3) construir unas pocas, pero grandes ideas de ciencia, ingeniería y/o las matemáticas.

Las problemáticas relevantes cientifica y socialmente son aquellas cuestiones de solución compleja y conflictiva donde se ponen en juego criterios e ideas científicas, así como aspectos políticos, económicos... que tienen implicaciones morales (Evagorou et al., 2012). Por ejemplo, la contaminación atmosférica o la edición genética. Las investigaciones en este sentido subrayan que trabajar estas cuestiones en el aula tiene un gran potencial para el desarrollo de habilidades y destrezas científicas clave del alumnado, por ejemplo el análisis crítico o la toma de decisiones en base a pruebas (Evagorou et al., 2012); el fomento de la actuación en el entorno (Reis, 2014); y la mejora de sus ideas científicas (Solé et al., 2020).

Por otro lado, el enfoque de la práctica científica, reconocido tanto nacional como internacionalmente (Crujeiras y Jiménez-Aleixandre, 2012; NRC, 2012), enfatiza la necesidad de involucrar al alumnado desde infantil en maneras de hacer, pensar, hablar y sentir análogas a las de la ciencia (Izquierdo, 2014). Es decir, en los procesos de modelizar (construir, usar y evaluar modelos), indagar (recoger y analizar pruebas a partir de la observación y/o experimentación) y argumentar (evaluar pruebas y construir argumentos). Desde esta perspectiva, el reto es encontrar contextos relevantes social y científicamente que involucren al alumnado de forma auténtica y genuina en estas prácticas. Es decir, partiendo de un contexto o actividad problemático cuya resolución sea percibida como desconocida y pertinente para el alumnado (Izquierdo, 2017).

Además, en didáctica de las ciencias existe consenso sobre la necesidad de partir de las ideas previas del alumnado (Driver et al., 1994) para, a continuación, construir unas pocas pero muy relevantes ideas de y sobre ciencia (Harlen, 2010; NRC, 2012). Desde esta perspectiva, el diseño de las SEA debe fomentar la evolución de las ideas del alumnado, a partir de expresarlas, evaluarlas y revisarlas, de forma que se profundicen y complejicen para acercarse a las ideas de la ciencia escolar objeto de aprendizaje (Couso, 2020; Izquierdo, 2014).

Sin embargo, la concreción de estos tres aspectos en una SEA es un reto para los docentes (Couso, 2014) especialmente en la etapa de primaria. Por ello, este articulo busca transparentar y ejemplificar el proceso de toma de decisiones relacionadas con el diseño 
e implementación de una SEA sobre un problema ambiental (la reducción de la contaminación del aire) que involucra a alumnado de 5o y 6o curso de primaria (10-12 años) en actividades de indagación y modelización. Además, se incluyen aspectos relevantes para su implementación en el aula (andamiaje y retroalimentación docente), así como ejemplos de las respuestas más comunes del alumnado.

\section{Características principales de nuestra SEA}

El diseño de SEA construidas iterativamente a partir de la incorporación de resultados de investigación es considerada una actividad clave (Guisasola et al., 2021). Desde esta perspectiva, es esencial que las propuestas expliciten los principios de diseño que las rigen, por ello, detallamos seguidamente los que caracterizan nuestra SEA.

\section{La contaminación como problemática social científicamente relevante}

Una de las problemáticas medioambientales más importantes actualmente en las grandes ciudades con consecuencias para la salud de los humanos y alteraciones en los ciclos biogeoquímicos es la contaminación atmosférica (Basagaña, 2018). Por ello, en las últimas décadas ha aumentado la preocupación científica y social por esta problemática y la búsqueda de soluciones. Esto ha quedado recogido por diversas organizaciones como la UNESCO (2017), que lo incluye dentro del ODS 11; o la OCDE (2020) que lo ha identificado como temática especialmente relevante para la promoción de la alfabetización científica para la ciudadanía responsable.

Actualmente no existe un consenso sobre las soluciones a esta problemática. Algunas propuestas hechas hasta el momento (p.ej. disminuir el tráfico rodado o aumentar las zonas verdes) a menudo chocan con intereses y/o maneras de vivir tanto de los individuos (p.ej. dificultad para acceder a algunos lugares) como de algunas grandes industrias.

Así, la búsqueda de soluciones a esta problemática pasa por la alfabetización de la ciudadanía para su actuación informada sobre el problema (Reis, 2014). Para ello, es imprescindible que estos comprendan algunas de las ideas del modelo corpuscular de la materia (p.ej. la idea de partícula en la mesoescala) y también, que reflexionen en torno a los valores, actitudes y creencias sociales que hay tras esta problemática y sus posibles soluciones (Solé et al., 2020).

\section{La indagación basada en la modelización como práctica científica auténtica}

De acuerdo con Reis (2014) una estrategia privilegiada para incorporar las controversias socio-científicas en el aula es la indagación. Es decir, involucrar al alumnado en el planteamiento de preguntas investigables y la búsqueda de pruebas para responderlas. Sin embargo, tal como enfatiza el marco de la indagación basada en la modelización (IBM), estas prácticas deben ir acompañadas de la construcción de las ideas científicas escolares clave (Couso, 2014).

Concretamente, el marco IBM busca superar algunas visiones ingenuas de las prácticas de indagación y modelización involucrando al alumnado en la exploración de fenómenos para construir y/o reconstruir las propias ideas a la luz de los resultados y el análisis obtenidos de la indagación (Schwarz y Gwekwerere, 2007). Para ello, se plantea involucrar al alumnado en prácticas como: expresar hipótesis en base a los propios modelos, discutir diseños de búsqueda de evidencias, etc. (Windschitl et al., 2008).

Teniendo en cuenta este marco y las propuestas de ciclos de modelización (Couso, 2020) e indagación (Jiménez-Liso, 2020) se han definido y secuenciado las diferentes habilidades 
y/o destrezas que se van a trabajar en cada momento de la SEA (ver Figura 1 más adelante). La SEA se inicia con el planteamiento de la problemática y la expresión de las ideas iniciales del alumnado. A continuación, estas se ponen a prueba a partir del diseño y ejecución de un proceso de búsqueda de pruebas: planteamiento de preguntas, recopilación y tratamiento de datos, etc. Finalmente, con las pruebas recogidas y las aportaciones de otras fuentes (p.ej. expertos) se construye un modelo final consensuado el cual, por un lado, es usado para proponer y justificar diferentes soluciones a la problemática y, por otro lado, puede ser aplicado y transferible a nuevas situaciones.

\section{Ideas previas e ideas clave del modelo materia y de la contaminación del aire}

La idea de corpuscularidad de la materia, es decir, entender la materia como discreta, es una idea clave tanto para la ciencia erudita como para la ciencia escolar ya que permite entender y actuar sobre multitud de fenómenos cotidianos (Harlen, 2010; NRC, 2012).

La investigación ha subrayado numerosas ideas alternativas sobre la materia, y especialmente sobre el aire, entre el alumnado de primaria. Algunos ejemplos son que el aire no es materia o que aire y oxígeno son la misma sustancia (Driver et al., 1994; Thornber et al., 1999). Por ello, es necesario proponer actividades que permitan ir superando progresivamente estas ideas y construyendo otras más cercanas al modelo corpuscular escolar esperado en primaria (Nebot y Márquez, 2019).

No obstante, existen pocas investigaciones en didáctica de las ciencias sobre las ideas que el alumnado de primaria tiene sobre la contaminación atmosférica. En algunos casos, se han identificado ideas alternativas como la confusión de la contaminación atmosférica con el efecto invernadero o con suciedad y residuos, por ejemplo, plásticos o basura (Iliopoulou, 2016; Thornber et al., 1999).

Sin embargo, Solé et al. (2020) identifican la contaminación atmosférica, y concretamente la idea de materia particulada en suspensión (PM por sus siglas en inglés), como relevante para las aulas ya que permite trabajar en profundidad aspectos del modelo corpuscular de la materia como la polisemia de la palabra partícula (que designa tanto partículas de polvo como de $\mathrm{O}_{2}$ ) y las diferentes escalas implicadas (macroescala, mesoescala y submicroescala).

Teniendo en cuenta todo esto se han definido dos ideas clave de la SEA para el alumnado de 5o y 6 o curso:

(1) El aire de nuestro entorno es materia. A nivel macroscópico este tiene unas propiedades (ocupa un volumen...) y a nivel meso/submicroscópico lo podemos imaginar como partes/partículas.

(2) La contaminación por PM es debida a la existencia en el aire de sustancias sólidas en suspensión, como polvo u hollín, las cuales no siempre se pueden ver a simple vista, pero sí usando una lupa binocular. Por el contrario, las partículas más pequeñas o submicroscópicas, como los átomos y/o moléculas que forman el aire (como el $\mathrm{O}_{2}$ ) no se pueden ver ni a simple vista ni con este instrumento.

\section{Descripción de las actividades clave de la SEA}

De manera iterativa durante dos cursos (2018-19 y 19-20) se ha diseñado, implementado y evaluado una SEA teniendo en cuenta los principios anteriores. La propuesta se ha implementado en más de 10 escuelas catalanas y ha contado con la participación de 647 alumnos de 50 y 60 curso de primaria (10-12 años) y sus respectivos docentes. 
La SEA final tiene una duración de 8 h y se compone por distintas actividades (figura 1). Todas ellas, así como la guía docente y otros recursos, se pueden consultar en abierto ${ }^{1}$. Sin embargo, se han identificado 3 actividades como las clave (destacadas en rojo en la figura 1) para hacer avanzar al alumnado en la construcción de las ideas y para involucrarlo en prácticas científicas: (1) la expresión en formato multimodal de las ideas iniciales y finales sobre aire limpio y contaminado, (2) el diseño y realización de una indagación genuina andamiada y (3) el planteamiento de acciones para disminuir la contaminación atmosférica justificadas en base a los resultados y aprendizajes.

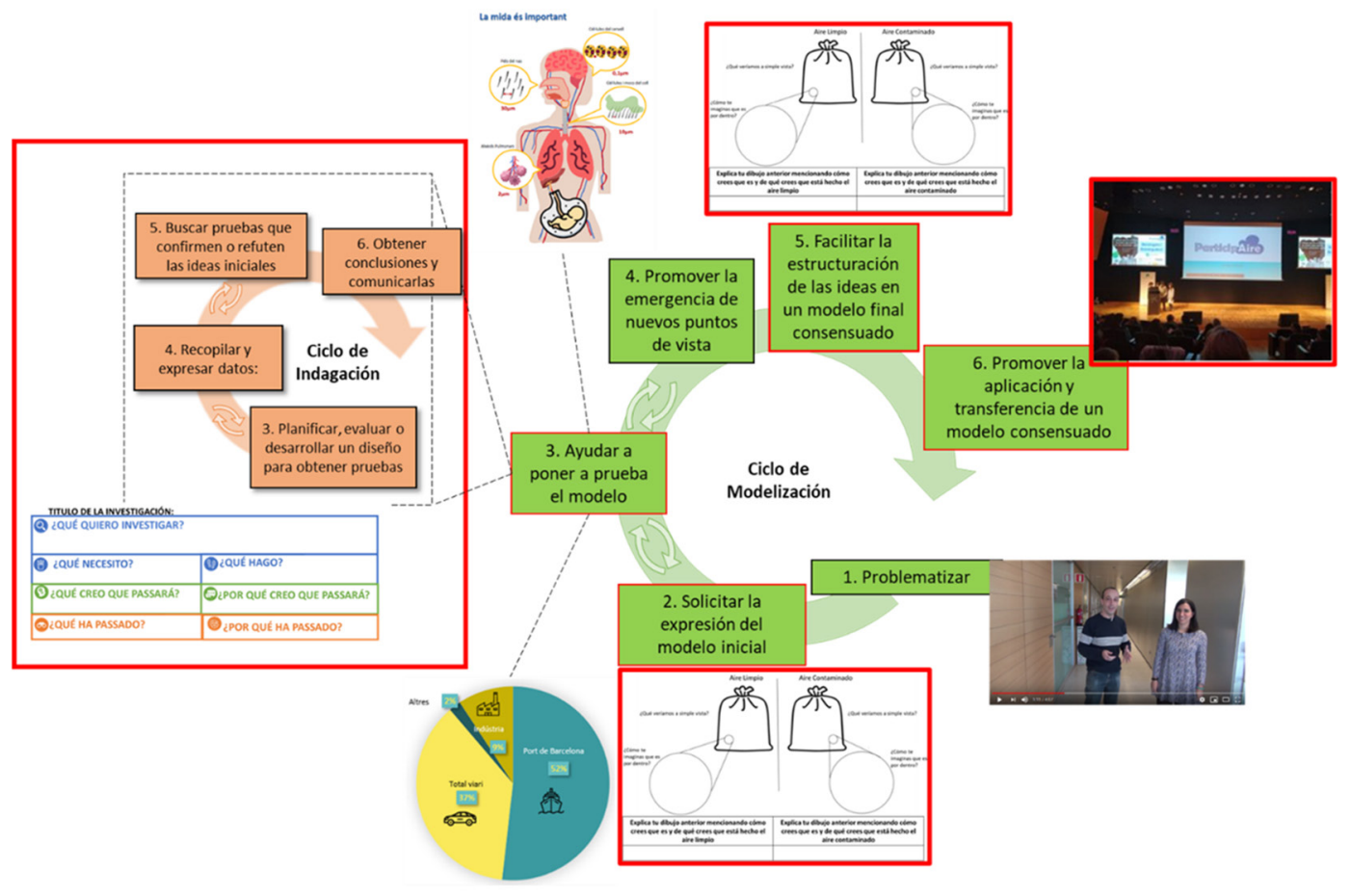

Figura 1. Esquema de la SEA. En él se muestran las fases del ciclo de modelización (en verde) y de indagación (en naranja) y su relación con las actividades de la propuesta. En rojo se destacan las actividades clave y las fases del ciclo a las que corresponden cada una de estas actividades.

\section{¿Cómo imaginamos el aire limpio y contaminado? Expresión multimodal de ideas}

Al inicio y final de la SEA el alumnado ha expresado de forma individual y multimodal (dibujando y explicando) cómo se imagina el aire limpio y contaminado tanto a simple vista (a escala macroscópica) como si lo pudiera ver por dentro (a escala meso/submicroscópica) (Figuras 2 y 3 ).

Ambas actividades tienen como objetivo que el alumnado exprese sus ideas sobre el modelo materia aplicado a dos fenómenos: aire limpio y aire contaminado.

La actividad de ideas iniciales (Tena y Couso, en prensa) nos ha permitido observar, por ejemplo, que la mayoría del alumnado tiene una idea del aire limpio como una sustancia continua y formada por un único componente al que llaman aire, oxígeno o viento (Figura 2).

1 https://ddd.uab.cat/record/225073?In=ca 
En el caso del aire contaminado, en cambio, las ideas iniciales sobre estructura y naturaleza son diversas. Además, encontramos alumnos que relacionan la contaminación con la presencia de virus, bacterias, $\mathrm{CO}_{2}$, o humo.

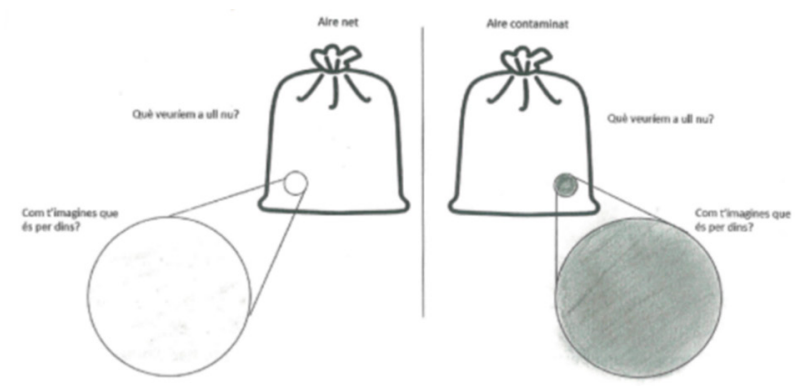

Aire limpio: Es un aire más o

Aire contaminado: He hecho menos limpio que creo que el aire contaminado negro es todo seguido porque creo que tiene humo

Figura 2 Ejemplo de producción inicial mayoritaria del alumnado.
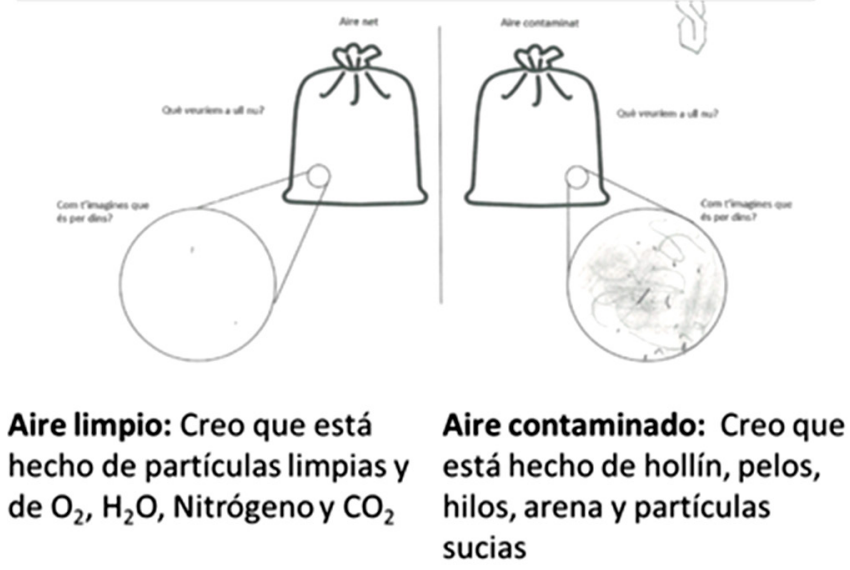

Figura 3. Ejemplo de producción final mayoritaria del alumnado.

El análisis de ideas finales nos ha permitido observar una evolución adecuada de las ideas del alumnado. La mayoría de las representaciones finales (Figura 3) hacen referencia a una sustancia continua con pequeñas "partículas" incrustadas en ella para describir tanto el aire limpio como contaminado. Además, en el aire limpio identifican más de un componente; y en el contaminado la mayoría representan partículas resultantes de la combustión además de hilos o polvo.

\section{¿Cómo podemos saber si el aire de nuestra escuela está contaminado? Diseño de una indagación genuina}

Para poner en conflicto y sofisticar sus ideas iniciales se ha involucrado al alumnado en un proceso genuino de indagación análogo al de los científicos. Para guiarlos en este proceso se les ha proporcionado la plantilla de andamiaje PaPER (Tena y Couso, 2020). Además, la retroalimentación docente también ha sido clave, especialmente en 3 momentos: (1) el diseño de la pregunta investigable, (2) el diseño de la metodología y (3) el análisis de los datos y elaboración de resultados y conclusiones. Algunos apoyos en los momentos anteriores, así como ejemplos de producciones iniciales y finales del alumnado han quedado recogidas en la tabla 1. 
Tabla 1. Ejemplos de producciones iniciales y finales del alumnado y andamiaje docente en 3 momentos clave

\begin{tabular}{|c|c|c|c|c|c|c|}
\hline & Propuestas iniciales alumnado & \multicolumn{4}{|l|}{ Ejemplos de andamiaje docente } & Propuestas finales alumnado \\
\hline \multirow{10}{*}{ 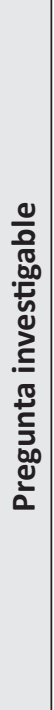 } & \multirow[t]{10}{*}{$\begin{array}{l}\text { “¿Qué grado de contaminación } \\
\text { hay en el patio?” (E1G6) }\end{array}$} & \multicolumn{4}{|c|}{$\begin{array}{l}\text { Criterios para fomentar la discusión e incorporación de modificaciones de las propias pre- } \\
\text { guntas del alumnado para hacerlas más investigables. }\end{array}$} & \multirow{10}{*}{$\begin{array}{l}\text { “¿Qué grado de contaminación } \\
\text { hay en la puerta principal y en la } \\
\text { puerta de detrás en } 24 \text { h y a un } \\
\text { metro y medio?” (E1G6) }\end{array}$} \\
\hline & & \multirow{2}{*}{ Criterio } & \multicolumn{2}{|c|}{ Si ha tenido en cuenta? } & \multirow{2}{*}{$\begin{array}{l}\text { ¿Cómo se puede } \\
\text { mejorar? }\end{array}$} & \\
\hline & & & Sí & NO & & \\
\hline & & $\begin{array}{l}\text { ¿La pregunta responde/aporta a la pregunta de inicial sobre como es la } \\
\text { calidad del aire de la escuela? }\end{array}$ & & & & \\
\hline & & $\begin{array}{l}\text { Se relacionan factores (cosas que queremos observar/medir) y variables } \\
\text { (cosas que son diferentes en cada caso...)? }\end{array}$ & & & & \\
\hline & & $\begin{array}{l}\text { ¿Es una pregunta del tipo "cómo" (de que manera, en qué situaciones...) } \\
\text { en vez de una pregunta de "por qué"? }\end{array}$ & & & & \\
\hline & & $\begin{array}{l}\text { ¿Es concreta (nos podemos imaginar qué instrumentos se utilizaran, } \\
\text { cuales serán los resultados...)? }\end{array}$ & & & & \\
\hline & & $\begin{array}{l}\text { ¿Es plausible (podemos dar respuesta con el material y condiciones que } \\
\text { tenemos)? }\end{array}$ & & & & \\
\hline & & No se puede responder con sí o no & & & & \\
\hline & & $\begin{array}{l}\text { La respuesta no se puede encontrar por internet, en un libro, no se puede } \\
\text { preguntar a la SIRI... }\end{array}$ & & & & \\
\hline \multirow{9}{*}{ 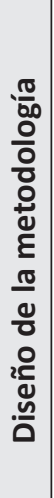 } & \multirow{9}{*}{$\begin{array}{l}\text { "Primero cogeríamos los mate- } \\
\text { riales, después engancharía- } \\
\text { mos el pegamento especial y } \\
\text { lo pondríamos en las dos puer- } \\
\text { tas y esperaríamos } 24 \mathrm{~h} \text { y si no } \\
\text { pasa nada cambiaríamos de } \\
\text { opinión." (E1G6) }\end{array}$} & \multicolumn{4}{|c|}{$\begin{array}{l}\text { Preguntas docentes para regular el proceso de diseño en función de las dificultades y nece- } \\
\text { sidades del grupo: }\end{array}$} & \multirow{9}{*}{$\begin{array}{l}\text { "Primero cogeríamos los materia- } \\
\text { les, después engancharíamos el } \\
\text { pegamento especial y lo pondría- } \\
\text { mos en las dos puertas y espe- } \\
\text { raríamos } 24 \mathrm{~h} \text { y si no pasa nada } \\
\text { cambiaríamos de opinión. medi- } \\
\text { ríamos las puertas y pondría- } \\
\text { mos las dos cartulinas a la misma } \\
\text { altura y así los dos están igual y } \\
\text { lo intentaríamos poner el mismo } \\
\text { tiempo." (E1G6) }\end{array}$} \\
\hline & & \multicolumn{4}{|l|}{ Concretar qué quieren medir } & \\
\hline & & \multicolumn{4}{|c|}{ ¿Qué queréis medir/observar?, ¿Cómo lo haréis para medirlo/observarlo? } & \\
\hline & & \multicolumn{4}{|c|}{ Identificar diferencias en la recogida de datos: } & \\
\hline & & \multirow{2}{*}{\multicolumn{4}{|c|}{$\begin{array}{l}\text { ¿Qué comparareis con qué? ¿Dónde haréis el experimento? ¿Cuándo lo haréis? } \\
\text { Concretar qué se debe mantener siempre igual y/o controlar }\end{array}$}} & \\
\hline & & & & & & \\
\hline & & \multicolumn{4}{|c|}{$\begin{array}{l}\text { ¿Qué cosas mirareis/ controlareis para que siempre sean iguales?,¿Pensáis que } \\
\text { puede influir la altura a la que recojamos la contaminación }\end{array}$} & \\
\hline & & \multicolumn{4}{|l|}{ Definir el análisis de los datos } & \\
\hline & & \multicolumn{4}{|c|}{$\begin{array}{l}\text { ¿Cómo lo haréis para observar/contar lo que recogeréis?, ¿Qué haréis con los datos } \\
\text { que obtengáis? }\end{array}$} & \\
\hline
\end{tabular}


Tabla 1. Continuación. Ejemplos de producciones iniciales y finales del alumnado y andamiaje hecho por el docente en tres momentos clave

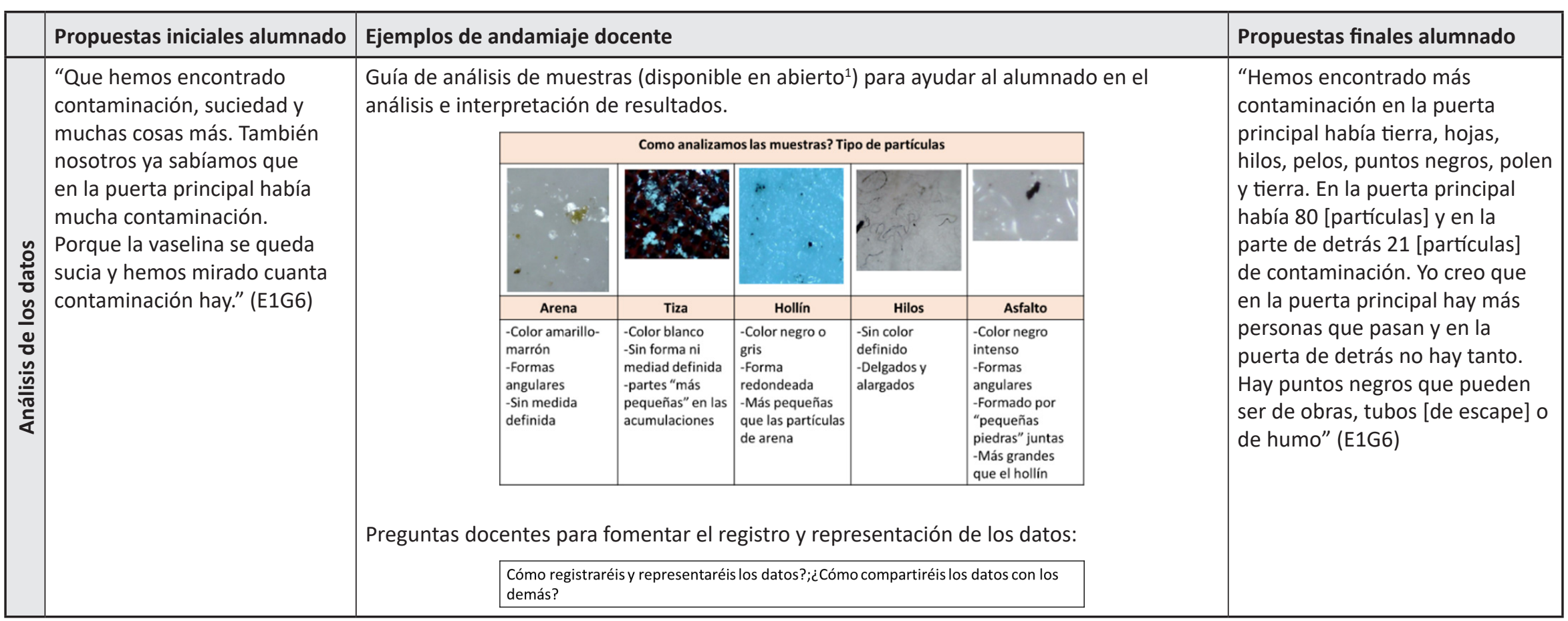




\section{¿Qué podemos hacer para mejorar la calidad del aire de la escuela? Planteamiento de soluciones justificadas}

Para acabar, el alumnado ha propuesto soluciones para reducir la contaminación en su escuela justificando porque son buenas acciones científica y socialmente. Para ello, han tenido que usar los resultados de las investigaciones y aprendizajes hechos a lo largo de la SEA.

Se ha observado un amplio abanico de propuestas (p.ej. plantar muros verticales, reducir el tráfico cerca de la escuela) que se han comunicado con formatos cercanos a los de la ciencia profesional (p.ej. presentaciones orales en congresos infantiles) y/o formatos artísticos o sociales (p.ej. videoclips, campañas de sensibilización) (Figura 4).
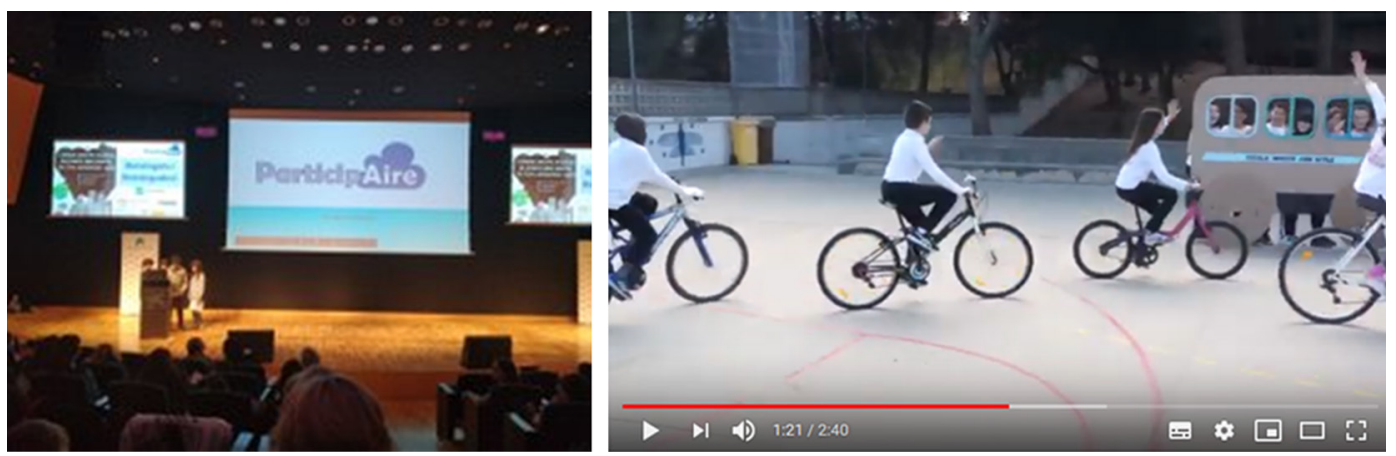

Figura 4. Acciones para mejorar la contaminación atmosférica en la escuela: un congreso infantil (izquierda) y un videoclip (derecha)

\section{Reflexiones finales}

Este artículo presenta un diseño de SEA enmarcada en un paradigma STEM con valores y que fomenta la participación del alumnado en prácticas científicas (Couso, 2017) usando el marco IBM y contextualizado en una controversia socio-científica. Estamos de acuerdo con Jiménez-Liso et al. (2021) en que el diseño e implementación de SEAs para las aulas basadas en investigación es un reto para docentes e investigadoras. Para ello, han sido necesarios diversos ciclos iterativos de mejora hasta llegar la versión final presentada.

La valoración final positiva hacia los aprendizajes construidos de ciencias (aire limpio y contaminación) y sobre ciencias (las maneras de hacer, pensar, hablar y sentir de la ciencia) así como los resultados presentados nos hacen pensar que la SEA promueve la evolución de las ideas de materia y las habilidades/destrezas de indagación del alumnado. Sin embargo, para poder estar seguros se pretende analizar en profundidad estos aspectos en futuras publicaciones (Tena y Couso, en prensa).

De acuerdo con García-Piqueras y Sotos-Serrano (2021) un aspecto clave para la implementación exitosa de SEA en el aula ha sido la formación y acompañamiento a los docentes. Esto ha implicado analizar y compartir las ideas a construir y las dificultades que presentan a los alumnos, así como la forma de andamiar y retroalimentar necesaria para promover indagación científica escolar genuina y autentica.

El hecho que la SEA se haya incorporada dentro de la programación habitual de algunas escuelas y se haya usado en diferentes eventos de formación docente nos hace pensar que esta puede ayudar a tender puentes entre la investigación en didáctica y las aulas de primaria. 


\section{Agradecimientos}

Esta investigación se ha realizado en el marco del doctorado en Educación de la UAB y con el apoyo de la Dra. Digna Couso. Ha sido financiada por el Ministerio de Ciencia, Innovación y Universidades (PGC2018-096581-B-C21) y la beca predoctoral FI-DGR-2018 dentro del grupo de investigación ACELEC (2017SGR1399).

\section{Bibliografía}

Basagaña, X. (2018). Els efectes de la contaminació en les persones: què en sabem i de què busquem evidències? Revista Ciències, 35, 28-34. DOI: https://doi.org/10.5565/ $\mathrm{rev} /$ ciencies.392

Couso, D. (2014). De la moda de "aprender indagando" a la indagación para modelizar: una reflexión crítica. En M.A. Héras, A. Lorca, B. Vázquez, A. Wamba, R. Jiménez. Investigación y transferencia para una educación en ciencias: Un reto emocionante (pp. 1-28). Huelva: Servicio de Publicaciones de la Universidad de Huelva. Recuperado de: http://uhu.es/26edce/actas/docs/conferencias/pdf/26ENCUENTRO_DCEConferenciaPlenarialnaugural.pdf

Couso, D. (2017). Por qué estamos en STEM? Un intento de definir la alfabetización STEM para todo el mundo y con valores. Revista Ciències, 34, 22. DOI: https://raco.cat/ index.php/Ciencies/article/view/338034

Couso, D. (2020). Aprender ciencia escolar implica construir modelos cada vez más sofisticados de los fenómenos del mundo. En D. Couso, M.R. Jimenez-Liso, C. Refojo y J.A. Sacristán (Coords), Enseñando Ciencia con Ciencia (pp. 63-74). FECYT \& Fundacion Lilly. Madrid: Penguin Random House. Recuperado de: https://www. fecyt.es/es/publicacion/ensenando-ciencia-con-ciencia

Crujeiras, B. y Jiménez-Aleixandre, M. P. (2012). Participar en las prácticas científicas. Alambique, 72, 12-19.

Driver, R., Squires, A., Rushworth, P. y Wood-Robinson, V. (1994). Part III: Materials and their properties. In Making sense of secondary science: Research into children's ideas (pp. 137-229). Routledge.

Evagorou, M., Jimenez-Aleixandre, M. P. y Osborne, J. (2012). "Should We Kill the Grey Squirrels?" A Study Exploring Students' Justifications and Decision-Making. International Journal of Science Education, 34(3), 401-428.

García-Piqueras, M. y Sotos-Serrano, M. (2021). Regeneración forestal tras un incendio: complejidad y protocolos en una aproximación STEM transversal. Revista Eureka Sobre Enseñanza Y Divulgación De Las Ciencias, 18(1), 1-19. DOI: https://doi. org/10.25267/Rev_Eureka_ensen_divulg_cienc.2021.v18.i1.1201

Guisasola, J., Ametller, J. y Zuza, K. (2021). Investigación basada en el diseño de Secuencias de Enseñanza-Aprendizaje: una línea de investigación emergente en Enseñanza de las Ciencias. Revista Eureka Sobre Enseñanza Y Divulgación De Las Ciencias, 18(1). DOI: https://doi.org/10.25267/Rev_Eureka_ensen_divulg_cienc.2021.v18.i1.1801

Harlen, W. (2010). Principles and big ideas of science education. Hatfield: Association for Science Education.

Iliopoulou, I. (2016). Can young students think systemically about the environment? The case of pollution. Education 3-13. DOI: https://doi.org/10.1080/03004279.2016.12 66688 
Izquierdo Aymeric, M. (2014). Los modelos teóricos en la "enseñanza de ciencias para todos" (eso, nivel secundario). Revista Bio-Grafía, 7(13), 69-85. DOI: https://doi. org/10.17227/20271034.13biografia69.85

Izquierdo, M. (2017). Atando cabos entre contexto, competencias y modelización. ¿Es posible enseñar ciencias a todas las personas? Modelling in Science Education and Learning, 10(1), 309-326.

Jiménez-Liso, M.R. (2020). Aprender ciencia escolar implica aprender a buscar pruebas para construir conocimiento (indagación). En D. Couso, M.R. Jimenez-Liso, C. Refojo y J.A. Sacristán (Coords), Enseñando Ciencia con Ciencia (pp. 53-62). FECYT \& Fundacion Lilly. Madrid: Penguin Random House. Recuperado de: https://www. fecyt.es/es/publicacion/ensenando-ciencia-con-ciencia

Jiménez-Liso, R., Delgado, L., Castillo-Hernández, F. J. y Baños, I. (2021). Contexto, indagación y modelización para movilizar explicaciones del alumnado de secundaria. Enseñanza de Las Ciencias, 39(1), 5-25.

Nebot Castelló, M. R. y Márquez Bargalló, C. (2019). El modelo cinético-corpuscular y las prácticas científicas: una propuesta basada en la dilatación térmica. Ápice. Revista de Educación Científica, 3(2), 21-35. DOI: https://doi.org/10.17979/ arec.2019.3.2.4625

NRC (2012). A Framework for K-12 Science Education: Practices, Crosscutting Concepts, and Core Ideas (Committee). The National Academies Press.

OCDE (2020). PISA 2024 Strategic Vision and Direction for Science (Issue March).

Reis, P. (2014). Acción Socio-Política sobre Cuestiones Socio-Científicas: Reconstruyendo la Formación Docente y el Currículo. Unipluriversidad, 14(2), 16-26.

Schwarz, C. V. y Gwekwerere, Y. N. (2007). Using a guided inquiry and modeling instructional framework (EIMA) to support preservice K-8 science teaching. Science Education, 91(1), 158.

Solé, C., Tena, È. y Couso, D. (2020). ¿Qué modelo de materia explica la contaminación? Alambique, 101, 30-36.

Tena, È. y Couso, D. (2020). ¿Cómo ayudar al alumnado a investigar en ciencias? Aula de Innovación Educativa, 298(Octubre), 15-20.

Tena, È. y Couso, D. (en prensa). What is city air made of? An analysis of pupils' conceptions of clean and polluted air. In Levrini et al. Engaging with Contemporary Challenges through Science Education Research. Springer

Thornber, J., Stanisstreet, M. y Boyes, E. (1999). School Students' Ideas about Air Pollution: Hindrance or Help for Learning ? Journal of Science Education and Technology, 8(1), 67-73.

UNESCO (2017). Educación para los Objetivos de Desarrollo Sostenible: Objetivos de aprendizaje. Recuperado de: https://unesdoc.unesco.org/ark:/48223/ pf0000252423

Windschitl, M., Thompson, J. y Braaten, M. (2008). Beyond the scientific method: Modelbased inquiry as a new paradigm of preference for school science investigations. Science Education, 92(5), 941-967. DOI: https://doi.org/10.1002/sce.20259 